\title{
Foreign Body in Stomach
}

National Cancer Institute

\section{Source}

National Cancer Institute. Foreign Body in Stomach. NCI Thesaurus. Code C35251.

Extraneous material located within the stomach. 